Polymer Journal, Vol. 3, No. 3, pp 357-364 (1972)

\title{
Determination of Mean Square Displacements of Polyethylene Molecules in Crystal Lattice
}

\author{
Koh-ichi Iohara, Kiyohisa Imada, and Motowo TaKayanagi \\ Department of Applied Chemistry, Faculty of Engineering, \\ Kyushu University, Fukuoka, Japan.
}

(Received September 13, 1971)

\begin{abstract}
X-ray scattering intensities of bulk crystallized polyethylene were measured at temperatures from $-165^{\circ} \mathrm{C}$ to $120^{\circ} \mathrm{C}$. Mean square displacements of carbon atoms from their average locations were represented by the symmetric tensor $\mathbf{U}$ having four independent elements, $U_{a a}, U_{b b}, U_{c c}$, and $U_{a b}$. These elements and the atomic coordinates were determined as a function of temperature by the least square method. The displacement of the carbon atom was a maximum in the direction perpendicular to the skeletal zigzag plane. The minimum displacement was found in the direction along the the molecular axis. The mean square displacements of atoms were separated into contributions from the molecular vibration and the lattice imperfection by extrapolating the elements of tensor $\mathbf{U}$ to the temperature of absolute zero. The amplitudes of molecular vibration obtained by X-ray measurements agreed fairly well with the theoretical values calculated on the assumption of the harmonic oscillator model by Kitagawa and Miyazawa at the temperatures below $0^{\circ} \mathrm{C}$. The atomic coordinates did not change below $0^{\circ} \mathrm{C}$. The skeletal plane of polyethylene molecule tended to deflect slightly toward the $a$ axis above $0^{\circ} \mathrm{C}$ with increasing temperature.

KEY WORDS X-rays / Atomic Coordinate / Atomic Displacement

/ Vibrational Amplitude / Lattice Imperfection / Anharmonicity /

Polyethylene /
\end{abstract}

Mean square amplitudes of molecular vibrations in polyethylene crystals have been studied by several authors, ${ }^{1-5}$ who determined the temperature factors related to the X-ray diffraction intensity. Kilian ${ }^{2,3}$ determined the temperature factor by using Ruland's method, ${ }^{6}$ showing the increase of vibration amplitudes of molecules with increasing temperature. He indicated that the observed temperature factor is the sum of the terms due to molecular vibration and to statistical lattice imperfection, and that the two terms could be separated by extrapolating the temperature factor to absolute zero. However, since the anisotropy of the temperature factor was not considered by him, the information concerning the direction of atomic displacements was not obtained. Aoki, et al. ${ }^{4}$ took into account the anisotropy of atomic displacements of molecules and determined the mean square displacements along the $a, b$ and $c$ axes by using the integrated intensities of three Bragg reflections. They compared their results with the theoretical temperature factors calculated by Kitagawa and Miyazawa, ${ }^{7}$ and indicated that the molecular vibration was anharmonic above $0^{\circ} \mathrm{C}$. However, the values of observed vibration amplitudes were not the absolute ones but shifted conveniently to fit the theoretical values. The variation of the atomic coordinates was not considered in their treatment. These parameters are however expected to have a considerable effect on the intensities of Bragg reflections. Kavesh and Schultz ${ }^{5}$ determined the atomic coordinates and the temperature factors using the integrated intensities of ten Bragg reflections. They indicated that the plane of the zigzag chain tends to deflect slightly toward the $a$ axis with increasing temperature. However, since the temperature factors were approximated by them using only two parameters, $K_{A B}$ and $K_{C}$ (the factors in the 
directions perpendicular and parallel to the molecular axis respectively), they could not produce detailed information on the directions of the atomic displacements.

In order to clarify the molecular motions in the crystal lattice, it is necessary to obtain more detailed information concerning the directions and the amplitudes of molecular vibrations. For this purpose, it is useful to represent the temperature factors by the tensor ellipsoid as proposed by Cruickshank. ${ }^{8}$ It is intended in this paper to determine all of the parameters including the atomic coordinates and the elements of the temperature factor tensor for a polyethylene crystal as a function of temperature. The directions and amplitudes of thermal vibrations of molecules will be discussed as well as the harmonicity of the thermal vibration as compared with the theoretical value. ${ }^{7}$

\section{THEORETICAL}

The structure factor of the unit cell for the $(h k l)$ reflection is defined by

$$
F(h k l)=\sum_{r} f_{r} D_{r} \exp \left\{-2 \pi i\left(h x_{r}+k y_{r}+l z_{r}\right)\right\}
$$

where $f_{r}$ is the scattering factor of the $r$-th atom and $x_{r}, y_{r}$, and $z_{r}$ are the fractional coordinates of that atom. If $X_{r}, Y_{r}$, and $Z_{r}$ are the component displacements of the $r$-th atom from the unit cell origin in the $a, b$, and $c$ lattice directions measured in Angstroms respectively, then $x_{r}, y_{r}$, and $z_{r}$ are defined as

$$
x_{r}=\frac{X_{r}}{a} \quad y_{r}=\frac{Y_{r}}{b} \quad z_{r}=\frac{Z_{r}}{c}
$$

The summation in eq 1 extends to all atoms in the unit cell. $D_{r}$ represents the Debye-Waller factor which is a smearing function caused by displacements of atoms, and is given by

$$
D_{r}=\exp \left\{-\frac{1}{4}\left(\mathbf{s} \cdot \mathbf{B}_{r} \cdot \tilde{\mathbf{s}}\right)\right\}
$$

where $\mathbf{s}$ is the reciprocal lattice vector, which is written as

$$
\mathbf{s}=\left(\frac{h}{a}, \frac{k}{b}, \frac{l}{c}\right)
$$

$\mathbf{B}_{r}$ is the temperature factor and is represented by the symmetric tensor having six independent elements. $^{8}$

$$
\mathbf{B}_{r}=\left(\begin{array}{r}
\boldsymbol{B}_{a a, r} \boldsymbol{B}_{a b, r} \boldsymbol{B}_{a c, r} \\
\boldsymbol{B}_{b b, r} \boldsymbol{B}_{b c, r} \\
\boldsymbol{B}_{c c, r}
\end{array}\right)
$$

When atomic displacements are harmonic or small enough, $\mathbf{B}_{r}$ is related to the tensor $\mathbf{U}_{r}$, which gives the amplitude of atomic displacement, as follows.

$$
\mathbf{B}_{r}=8 \pi^{2} \mathbf{U}_{r}=8 \pi^{2}\left(\begin{array}{r}
U_{a a, r} U_{a b, r} U_{a c, r} \\
U_{b b, r} U_{b c, r} \\
U_{c c, r}
\end{array}\right)
$$

The mean square displacement in the direction specified by the unit vector $\mathbf{p}=\left(p_{a}, p_{b}, p_{c}\right)$ is written as $^{8}$

$$
\left\langle u^{2}\right\rangle_{r}=\sum_{i} \sum_{j} U_{i j, r} p_{i} p_{j} \quad(i, j=a, b, c)
$$

Figure 1 shows the crystal structure and the symmetry elements of polyethylene determined
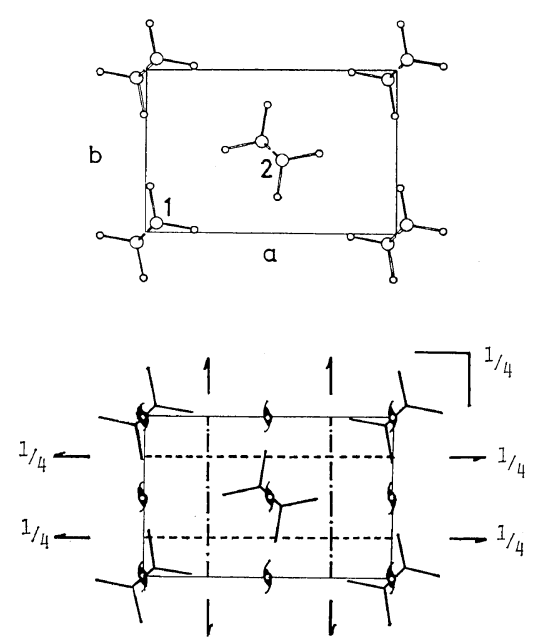

Figure 1. Structure and symmetry elements of a polyethylene crystal.

by Bunn. ${ }^{1}$ Polyethylene crystals belong to the orthorhombic system having four methylene groups in the unit cell. X-ray scattering by hydrogen atoms were neglected because the $\mathrm{X}$ ray scattering power of the hydrogen atoms is much smaller than that of carbon atoms. ${ }^{9}$ Since the polyethylene crystal is centro-symmetric, the summation in eq 1 is done for the carbon atom-1 located at $(x, y, z)$ and the carbon atom- 


\section{Displacements of Polyethylene Molecules in Crystals}

2 at $[(1 / 2)+x,(1 / 2)-y, z]$. The crystal has a symmetry of mirror plane parallel to $a b$ plane at $z=1 / 4$. Therefore, the following parameters are determined a priori.

$$
z=\frac{1}{4} \quad U_{a c, r}=U_{b c, r}=0 \quad(r=1,2)
$$

The crystal has a symmetry of glide plane $a$ parallel to $a c$ plane at $y=1 / 4$. Therefore, the following parameters are related with each other.

$$
\begin{aligned}
U_{a a, 1}=U_{a a, 2} \equiv U_{a a} & U_{b b, 1}=U_{b b, 2} \equiv U_{b b} \\
U_{c c, 1}=U_{c c, 2} \equiv U_{c c} & U_{a b, 1}=-U_{a b, 2} \equiv U_{a b}
\end{aligned}
$$

By taking into account eq $3,4,6,8$, and 9 , eq 1 is rewritten as follows.

$$
\begin{aligned}
F(h k l)= & 2 f\left[\operatorname { e x p } \left\{-2 \pi^{2}\left(U_{a a}\left(\frac{h}{a}\right)^{2}+U_{b b}\left(\frac{k}{b}\right)^{2}\right.\right.\right. \\
& \left.\left.+U_{c c}\left(\frac{l}{c}\right)^{2}+2 U_{a b} \frac{h}{a} \frac{k}{b}\right)\right\} \\
& \times \cos 2 \pi\left(h x+k y+\frac{l}{4}\right) \\
& +(-1)^{h+k} \exp \left\{-2 \pi^{2}\left(U_{a a}\left(\frac{h}{a}\right)^{2}\right.\right. \\
& +U_{b b}\left(\frac{k}{b}\right)^{2}+U_{c c}\left(\frac{1}{c}\right)^{2} \\
& \left.\left.\left.-2 U_{a b} \frac{h}{a} \frac{k}{b}\right)\right\} \cos 2 \pi\left(h x-k y+\frac{l}{4}\right)\right]
\end{aligned}
$$

Thus, the structure factor of the polyethylene unit cell is represented by eq 10 , which includes the atomic coordinates parameters, $x$ and $y$, and the atomic displacement parameters, $U_{a a}$, $U_{b b}, U_{c c}$ and $U_{a b}$.

\section{EXPERIMENTAL}

\section{Sample Preparation}

The sample used in this work is a commercial grade high-density linear polyethylene, Hizex $1200 \mathrm{~J}$, the product of Mitsui Petro-Chemical Industries Co., Ltd. Its molecular weight $\left(\mathbf{M}_{\eta}\right)$ was 30800 , melt index was 9.2, and the degree of branching was $2 \mathrm{CH}_{3} / 1000 \mathrm{CH}_{2}$. The sample was melted at $180^{\circ} \mathrm{C}$ and cooled to room temperature at the rate of $0.1^{\circ} \mathrm{C} / \mathrm{min}$. This bulkcrystallized sample was then annealed at $120^{\circ} \mathrm{C}$ for ten hours. No preferred orientation of crystal planes was found by checking with the measurement of orientation function.

\section{$X$-ray Scattering Measurement}

$X$-ray scattering intensities were measured by the reflection method using an X-ray diffractometer with Ni-filtered $\mathrm{Cu}-\mathrm{K} \alpha$ radiation and a Geiger-Müller (GM) counter. The intensity of the incident beam was regulated so as to make its maximum on the GM counter less than 100 counts per second. Count miss of the GM counter was found to be less than $1.5 \%$.

The specimen was attached to the Rigakudenki Company low temperature device. The scattering intensities were measured over the temperature range from $-165^{\circ} \mathrm{C}$ to $120^{\circ} \mathrm{C}$.

\section{Method of Data Analysis}

The parameters in eq 10 including the atomic coordinates and the elements of tensor $\mathbf{U}$ were determined according to the least squares method, ${ }^{10}$ which is generally used for a precise determination of crystal structure. The most probable values of these parameters are assumed to minimize the value of $R$ defined by the following equation.

$$
R \equiv \sum_{h k l} w(h k l)\left\{\left|F_{\mathrm{o}}(h k l)\right|-K\left|F_{\mathrm{c}}(h k l)\right|\right\}^{2}
$$

where $F_{\mathrm{o}}$ and $F_{\mathrm{c}}$ are the observed and calculated structure factors respectively, $K$ is the scale factor and $w(h k l)$ is the weight of $(h k l)$ reflection.

The integrated intensities of 32 reflections were used. Reflections partly super-imposed were separated by the ratio of peak intensities. Reflections completely super-imposed were separated by the ratio of calculated intensities. After the corrections of the intensity data were made according to the conventional procedure ${ }^{10}$ the observed structure factors, $F_{0}(h k l)$, were determined.

Table I lists the 32 crystal planes, the integrated intensities, and the weights employed at $28^{\circ} \mathrm{C}$. As the weights of (110) and (200) reflections with strong intensities, the values of 0.2 and 0.5 were assigned respectively. ${ }^{10}$ The weights of superposed reflections were set to $1.0 / n$, where $n$ denotes the number of superposition.

Calculations by the least square method were made by using the electronic computor, FACOM 230-60, in the Computor Center of Kyushu University. The atomic coordinates and the 
Table I. Crystal planes, integrated intensities and weights used in the least square method at $28^{\circ} \mathrm{C}$

\begin{tabular}{|c|c|c|}
\hline$(h k l)$ & Intensity & Weight \\
\hline 110 & 1000.0 & 0.2 \\
\hline 200 & 316.2 & 0.5 \\
\hline 210 & 16.4 & 1.0 \\
\hline 020 & 35.7 & 1.0 \\
\hline 120 & 9.3 & 1.0 \\
\hline 011 & 39.8 & 1.0 \\
\hline 310 & 31.7 & 1.0 \\
\hline 111 & 27.1 & 1.0 \\
\hline 201 & 33.3 & 1.0 \\
\hline 220 & 13.6 & 1.0 \\
\hline 211 & 19.1 & 1.0 \\
\hline 400 & 6.5 & 1.0 \\
\hline 410 & 3.4 & 0.3 \\
\hline 320 & 7.5 & 0.3 \\
\hline 121 & 21.7 & 0.3 \\
\hline 311 & 17.3 & 1.0 \\
\hline 221 & 6.0 & 0.5 \\
\hline 130 & 3.3 & 0.5 \\
\hline 230 & 1.7 & 0.3 \\
\hline 401 & 6.0 & 0.3 \\
\hline 420 & 1.4 & 0.3 \\
\hline 411 & 0.8 & 0.3 \\
\hline 321 & 4.7 & 0.3 \\
\hline 510 & 0.9 & 0.3 \\
\hline 031 & 5.3 & 0.5 \\
\hline 330 & 0.4 & 0.5 \\
\hline 231 & 5.4 & 0.3 \\
\hline 520 & 1.6 & 0.3 \\
\hline 002 & 6.9 & 0.3 \\
\hline 511 & 7.8 & 0.3 \\
\hline 112 & 7.9 & 0.3 \\
\hline 202 & 6.6 & 0.3 \\
\hline
\end{tabular}

isotropic temperature factor reported by Bunn ${ }^{1}$ were used as the starting values of the parameters. Thus, the most probable values of $x$, $y, U_{a a}, U_{b b}, U_{c c}$, and $U_{a b}$ in eq 10 were determined.

\section{RESULTS}

Table II lists the atomic coordinates, $x$ and $y$ (fractional coordinates), and their standard deviations, $\sigma(x)$ and $\sigma(y)$, at temperatures from $-165^{\circ} \mathrm{C}$ to $120^{\circ} \mathrm{C}$. Figure 2 shows the temperature dependence of the atomic coordinates, $X$ and $Y$ (Angstrom unit), and the setting angle,
Table II. Fractional coordinates of carbon atom, $x$ and $y$

\begin{tabular}{rcc}
\hline Temp, ${ }^{\circ} \mathrm{C}$ & $x \pm \sigma(x)$ & $y \pm \sigma(y)$ \\
\hline-165 & $0.042 \pm 0.003$ & $0.070 \pm 0.004$ \\
-115 & $0.042 \pm 0.002$ & $0.070 \pm 0.004$ \\
-60 & $0.042 \pm 0.002$ & $0.070 \pm 0.004$ \\
-2 & $0.042 \pm 0.002$ & $0.070 \pm 0.003$ \\
28 & $0.043 \pm 0.002$ & $0.069 \pm 0.003$ \\
60 & $0.044 \pm 0.003$ & $0.067 \pm 0.004$ \\
93 & $0.043 \pm 0.003$ & $0.068 \pm 0.004$ \\
120 & $0.045 \pm 0.003$ & $0.066 \pm 0.005$ \\
\hline
\end{tabular}

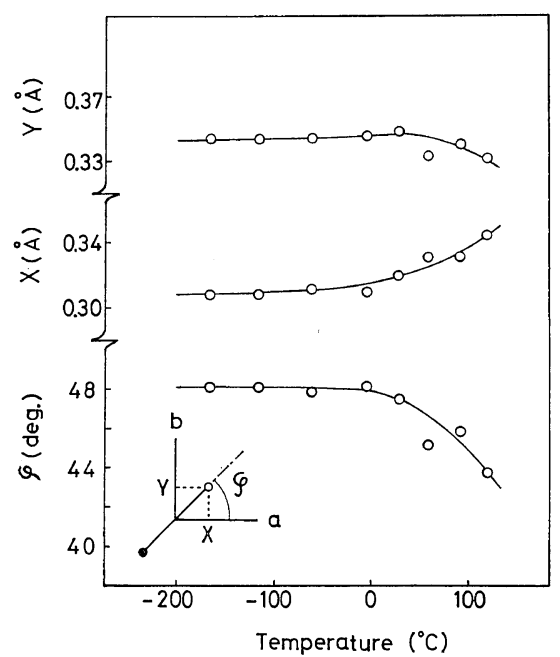

Figure 2. Atomic coordinates $X, Y$ and setting angle $\varphi v s$. temperature.

$\varphi$, between the $a$ axis and the skeletal zigzag plane. As seen in Figure 2, $X, Y$ and $\varphi$ do not change below $0^{\circ} \mathrm{C}$. On the other hand, increase in $X$ and decreases in $Y$ and $\varphi$ are seen with increasing temperature in the temperature region above $0^{\circ} \mathrm{C}$. These facts show that the skeletal zigzag plane of polyethylene molecule tends to deflect toward the $a$ axis with increasing temperature. These observations agree with those reported by Kasai and Kakudo, ${ }^{11}$ and Kavesh and Schultz. ${ }^{5}$

Table III lists the elements of the tensor $\mathbf{U}$ and their standard deviations at the temperatures from $-165^{\circ} \mathrm{C}$ to $120^{\circ} \mathrm{C}$. Figure 3 shows the temperature dependence of $U_{a a}, U_{b b}, U_{c c}$, and $-U_{a b}$. Here, $U_{a a}, U_{b b}$ and $U_{c c}$ indicate the mean square displacements along the $a, b$, and 
Displacements of Polyethylene Molecules in Crystals

Table III. Values of $U_{i j}$ in $10^{-2} \AA^{2}$

\begin{tabular}{rcccc}
\hline Temp, ${ }^{\circ} \mathrm{C}$ & $U_{a a} \pm \sigma\left(U_{a a}\right)$ & $U_{b b} \pm \sigma\left(U_{b b}\right)$ & $U_{c c} \pm \sigma\left(U_{c c}\right)$ & $U_{a b} \pm \sigma\left(U_{a b}\right)$ \\
\hline-165 & $5.5 \pm 1.9$ & $5.7 \pm 1.8$ & $1.9 \pm 1.0$ & $-0.6 \pm 1.3$ \\
-115 & $6.2 \pm 1.9$ & $6.1 \pm 1.8$ & $1.6 \pm 1.1$ & $-0.4 \pm 1.3$ \\
-60 & $6.8 \pm 2.0$ & $7.6 \pm 1.9$ & $1.3 \pm 1.1$ & $-0.8 \pm 1.4$ \\
-2 & $7.9 \pm 1.9$ & $8.8 \pm 1.8$ & $1.2 \pm 1.0$ & $-1.0 \pm 1.4$ \\
28 & $9.2 \pm 1.9$ & $10.4 \pm 1.9$ & $1.7 \pm 1.0$ & $-0.1 \pm 1.3$ \\
60 & $10.0 \pm 2.4$ & $8.4 \pm 2.0$ & $1.5 \pm 1.1$ & $-1.2 \pm 1.6$ \\
93 & $10.8 \pm 2.5$ & $10.2 \pm 2.3$ & $3.0 \pm 1.3$ & $-0.5 \pm 1.6$ \\
120 & $11.8 \pm 2.9$ & $9.5 \pm 2.4$ & $2.9 \pm 1.4$ & $-1.0 \pm 1.8$ \\
\hline
\end{tabular}

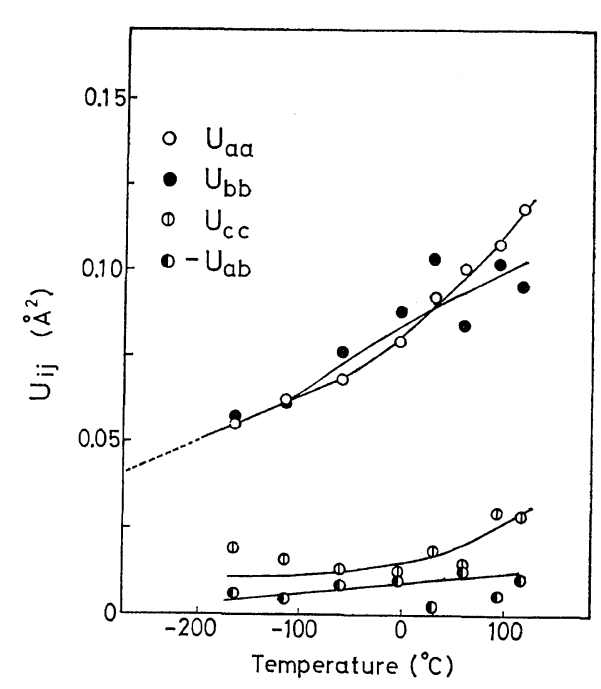

Figure 3. Elements of tensor $\mathbf{U} v s$. temperature.

$c$ axes, respectively. As seen in Figure 3, $U_{a a}$ is nearly equal to $U_{b b}$ and about five times as large as $U_{c c}$. In other words, the atomic displacements along the $a$ and $b$ axes are almost the same and much larger than that of $c$ axis as reported by Aoki, et al. ${ }^{4}$. The equality of the displacements along the $a$ and $b$ axes seems to be reasonable, as far as the setting angle of the skeletal plane to the $a$ axis is about $45^{\circ}$. The anisotropy of the molecular force field in the directions parallel and perpendicular to the molecular axis offers a reasonable explanation for the difference between the displacements along the $a$ and $c$ axes or $b$ and $c$ axes. The atoms are bonded by strong covalent bonds along the molecular axis compared with the rather weak intermolecular van der Waals bonds.

As shown in Table III, the cross element, $U_{a b}$, which has newly been introduced in this study, has a negative value. This result is closely related to the fact that the displacement of the carbon atom is maximum in the direction perpendicular to the skeletal zigzag plane if the tensor $\mathbf{U}$ is diagonalized according to the transformation method to the principal axes. Figure 4 shows the direction of the two principal axes ( $L A$ and $S A$ ) and the root mean square displacements of the carbon atom along these axes at $-115^{\circ} \mathrm{C}$. The value in parentheses in Figure 4 is the root mean square displacement along the $c$-axis, the third principal axis. The fact that the direction of $L A$ axis, that of the maximum

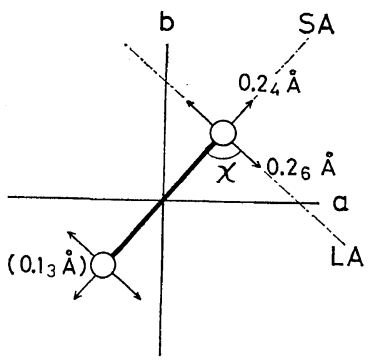

Figure 4. Root mean square displacements along the $L A, S A$ and molecular axis (parentheses) at $-115^{\circ} \mathrm{C}$.

Table IV. Values of $U_{L A}$, and $U_{S A}$ in $10^{-2} \AA^{2}$, and $\chi$ in degree

\begin{tabular}{rrrr}
\hline Temp, ${ }^{\circ} \mathrm{C}$ & $U_{L A} \pm \sigma\left(U_{L A}\right)$ & $U_{S A} \pm \sigma\left(U_{S A}\right)$ & $\chi \pm \sigma(\chi)$ \\
\hline-165 & $6.2 \pm 1.9$ & $5.0 \pm 1.8$ & $81 \pm 62$ \\
-115 & $6.6 \pm 1.9$ & $5.7 \pm 1.9$ & $90 \pm 85$ \\
-60 & $8.1 \pm 2.0$ & $6.3 \pm 1.9$ & $74 \pm 42$ \\
$-\quad 2$ & $9.4 \pm 1.9$ & $7.3 \pm 1.9$ & $75 \pm 35$ \\
60 & $10.7 \pm 2.5$ & $7.7 \pm 2.0$ & $105 \pm 30$ \\
93 & $11.1 \pm 2.5$ & $9 \cdot 9 \pm 2.2$ & $103 \pm 80$ \\
120 & $12.2 \pm 2.9$ & $9.1 \pm 2.3$ & $111 \pm 34$ \\
\hline
\end{tabular}


displacement, is almost perpendicular to the skeletal zigzag plane suggests that the rotational vibration of molecules around their axes will contribute considerably to the temperature factor as discussed in a later part.

Table IV lists the values of $U_{L A}, U_{S A}$, and $\chi$, and their standard deviations at various temperatures, where $U_{L A}$ and $U_{S A}$ denote the mean square displacements along the $L A$ and $S A$ axes, respectively, and $\chi$ is the angle between the $L A$ axis and the skeletal zigzag plane.

\section{DISCUSSION}

It is generally known that the atoms in crystal lattice vibrate harmonically in the low temperature region, and that the square of vibration amplitude of a harmonic oscillator is proportional to absolute temperature except in the temperature region near absolute zero, where the quantum effect must be considered. In the case of a polyethylene crystal, the quantum effect on the molecular vibrations which determine the temperature factor is considered to be negligible in the temperature region of this study. ${ }^{7}$ Then, extrapolation of $U_{i j}$ at the temperatures below $0^{\circ} \mathrm{C}$ should give zero value at absolute zero in Figure 3, if $U_{i j}$ shows the mean square displacements of atoms due only to the molecular vibrations. As seen in Figure 3, the observed values of the elements of the tensor $\mathbf{U}$ seem to decrease with decreasing temperature. However, extrapolation of the curves of $U_{i j}$, especially $U_{a a}$ and $U_{b b}$, to the temperature absolute zero gives considerably large values. This fact will be explained by taking into account the displacements of the carbon atoms caused by the lattice imperfections of the first kind as discussed by Kilian. ${ }^{3}$ In such a case, the displacements determined by $\mathrm{X}$-ray measurements are separated into two kinds of contributions from the molecular vibrations and the lattice imperfections. According to the convolution theory, DebyeWaller factor is written as follows. ${ }^{3}$

$$
\begin{aligned}
D= & \exp \left\{-2 \pi^{2}(\mathbf{s} \mathbf{U} \tilde{\mathbf{s}})\right\}=\exp \left\{-2 \pi^{2}\left(\mathbf{s}^{T} \tilde{\mathbf{s}}\right)\right\} \\
& \times \exp \left\{-2 \pi^{2}\left(\mathbf{s} \mathbf{U}^{0} \tilde{\mathbf{s}}\right)\right\}
\end{aligned}
$$

Thus,

$$
\mathbf{U}=\mathbf{U}^{T}+\mathbf{U}^{0}
$$

where $\mathbf{U}^{T}$ and $\mathbf{U}^{0}$ are the tensors giving the mean square amplitudes of molecular vibrations and the mean square displacements of atoms due to the lattice imperfections, respectively. According to eq $13, \mathbf{U}^{0}$ is obtained by extrapolation of the temperature dependence curve of $U_{i j}$ to the absolute zero.

The extent of lattice imperfection may be different between samples with different preparation histories. Crystallization conditions, annealing conditions, $\gamma$-ray irradiations, etc., will strongly influence the imperfections of the crystal lattice. In the case of the bulk crystal sample used in this study, the mean square displacements due to the lattice imperfections were determined as follows.

$$
U_{a a}^{0} \simeq U_{b b}^{0} \simeq 0.04 \AA^{2} \quad U_{a b}^{0} \simeq 0 \AA^{2}
$$

$U_{c c}^{0}$ could not be determined because $U_{c c}$ shows an unexplainable increase with decreasing temperature. In any way, $U_{c c}^{0}$ is considered to be much smaller than $U_{a a}^{0}$ or $U_{b b}^{0}$ according to Figure 3. These results indicate that the lattice imperfections are almost isotropic in the direction perpendicular to the molecular axis, whereas the imperfections along the molecular axis are small because of the strong intrachain force field.

Hereafter we assume that the imperfection of the crystal is frozen and unchanged with

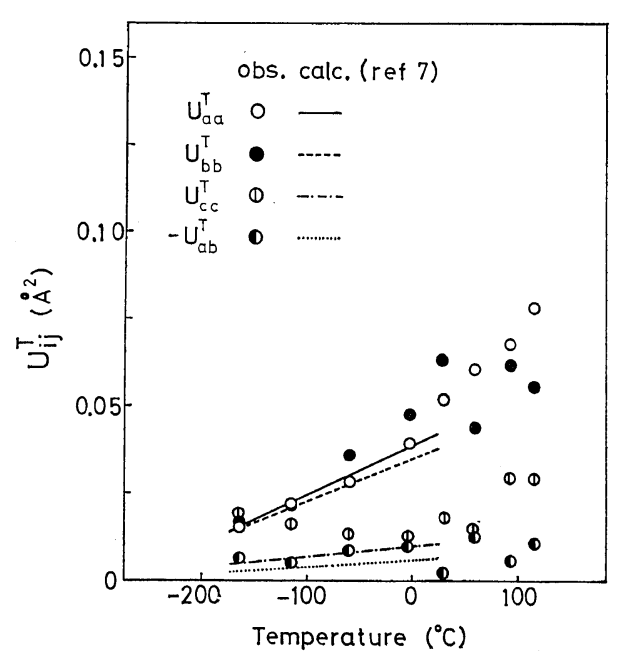

Figure 5. Elements of tensor $\mathbf{U}^{T} v s$. temperature. 
increasing temperature. This assumption is considered to be acceptable in the low temperature region as discussed later. Then, the elements $U_{i j}^{T}$ of the tensor $\mathbf{U}^{T}$, which give the mean square amplitudes of the molecular vibration can be determined by using eq 13 . Figure 5 shows the temperature dependence of $U_{i j}^{T}$. Here, $U_{c c}^{T}$ is assumed to be equal to $U_{c c}$ because $U_{c c}^{0}$ cannot be determined.

Kitagawa and Miyazawa ${ }^{7}$ have calculated the frequency distribution of the normal vibration in the polyethylene crystal and the amplitude of every vibrational mode on the basis of a harmonic oscillator model, and they derived the tensors of temperature factor of X-ray diffraction at $-173^{\circ} \mathrm{C}$ and $25^{\circ} \mathrm{C}$. Their results, which are converted to $U_{i j}^{T}$ by using the relation of eq 6 , are shown in Figure 5 by full line $\left(U_{a a}^{T}\right)$, broken line $\left(U_{b b}^{T}\right)$, chain line $\left(U_{c c}^{T}\right)$ and dotted line $\left(U_{a b}^{T}\right)$.

As seen in Figure 5, agreements between the elements of the tensors obtained by the X-ray measurements and the theoretical calculations are fairly good especially in the low temperature region below about $0^{\circ} \mathrm{C}$. In order to determine $U_{i j}^{T}$, the lattice imperfection is assumed not to vary with temperature as already mentioned. The agreements with the calculated values seem to suggest that the assumption is reasonable at least in the low temperature region. Futhermore, the agreements seem to support the fact that the polyethylene molecules in the crystal vibrate almost harmonically at these low temperatures.

In the temperature region above $0^{\circ} \mathrm{C}$, on the other hand, the values of $U_{i j}^{T}$ evaluated by $\mathrm{X}$ ray measurements increase rapidly with increasing temperature and show larger values than those of the theoretical $U_{i j}^{T}$. This fact can be ascribed to an increase of anharmonicity of molecular vibration as discussed by Aoki, et al. ${ }^{4}$.

The tensor $\mathbf{U}^{T}$ was diagonalized and the mean square amplitudes of molecular vibration along two principal axes of the tensor ellipsoid, $U_{L A}^{T}$ and $U_{S A}^{T}$, were determined. Figure 6 shows the temperature dependence of $U_{L A}^{T}$ and $U_{S A}^{T} . \quad U_{L A}^{T}$, which indicates the maximum mean square amplitude of molecular vibration, is found in a direction almost perpendicular to the skeletal zigzag plane, and $U_{S A}^{T}$, which shows the minimum mean square amplitude in $a b$ plane, is

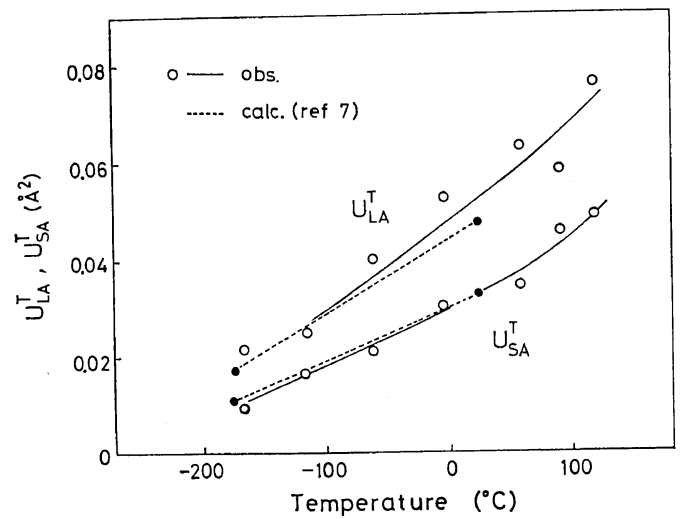

Figure 6. Mean square amplitudes along the $L A$ and $S A$ axes $v s$. temperature.

found in the direction including the skeletal plane and perpendicular to the molecular axis.

According to the calculation by Kitagawa and Miyazawa, ${ }^{7}$ the temperature factor is mainly determined by the intermolecular vibration modes with low frequencies as found in translational or rotational vibration of molecular chains and does not relate to the high frequency intramolecular vibrations. Then, $U_{L A}^{T}$ is considered to be mainly determined by the translational mode in the $L A$ direction as indicated in Figure $7 \mathrm{a}$ and the rotational mode around the molecular axis as indicated in Figure $7 \mathrm{~b}$, whilst $U_{S A}^{T}$ is dependent on the translational (a)

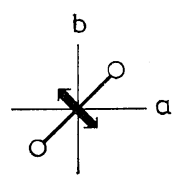

(b)

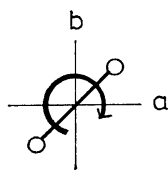

(c)

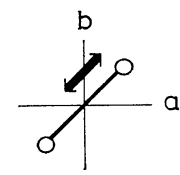

Figure 7. Vibration modes along the $L A$ and $S A$ axes: (a) translational mode along $L A$ axis; (b) rotational mode around molecular axis; (c) translational mode along $S A$ axis.

mode in the $S A$ direction as indicated in Figure $7 \mathrm{c}$.

In Figure 6, the theoretical values calculated on the assumption of harmonic oscillation ${ }^{7}$ (filled circles and broken lines) are compared with the values found based on the $\mathrm{X}$-ray measurements 
(open circles and full lines). The observed values of $U_{S A}^{T}$ agree fairly well with the theoretical ones until about $60^{\circ} \mathrm{C}$. This fact indicates that the translational mode of molecules in the $S A$ direction is harmonic up to relatively high temperatures. On the other hand, the observed values of $U_{L A}^{T}$ are larger than the calculated ones even at temperatures as low as $0^{\circ} \mathrm{C}$. If it is assumed that the harmonicity of the translational mode along the $L A$ direction is not very different from that along the $S A$ direction, the above fact leads to the conclusion that the rotational mode around the molecular axis becomes anharmonic more easily than the translational mode.

It is impossible to separate the amplitude of rotational vibration and that of translational vibration along the $L A$ axis from the observed $U_{L A}^{T}$ and criticize their harmonicity without any assumptions. The theoretical value of the ratio of the mean square amplitudes of these two vibration modes was estimated after the calculation of Kitagawa and Miyazawa. ${ }^{7}$ By using the theoretical ratio, the translational and rotational vibration amplitudes can be separated from the observed values of $U_{L A}^{T}$. The mean square amplitudes of rotational vibration, $\left\langle\omega^{2}\right\rangle$, evaluated based on this assumption are plotted against temperature in Figure 8.

As shown in Figure 2, the skeletal plane turns slightly toward the $a$ axis in the temperature region above $0^{\circ} \mathrm{C}$. Such a tendency is related to the anharmonic vibration of molecules above $0^{\circ} \mathrm{C}$.

The vibration amplitude along the molecular axis, $U_{c c}^{T}$, was not determined, because the extent of the lattice imperfection in this direction, $U_{c c}^{0}$, could not be estimated. Therefore, the comparison of the observed $U_{c c}^{T}$ with the theoretical values $^{7}$ was not made. The rapid increase in $U_{c c}$ at the temperatures above $60^{\circ} \mathrm{C}$ as shown in Figure 3 may be considered as an indication

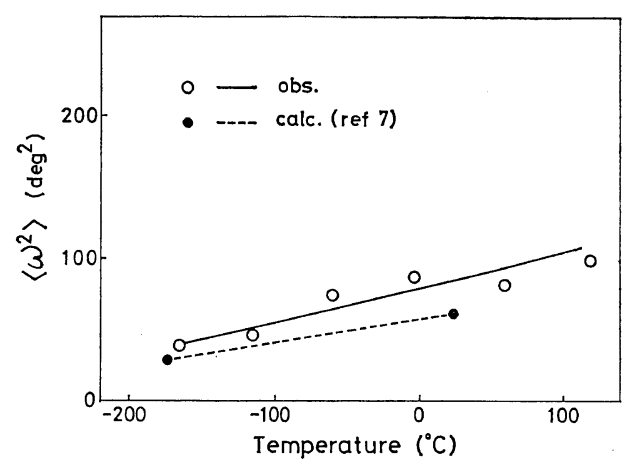

Figure 8. Mean square amplitude of rotational vibration $\left\langle\omega^{2}\right\rangle v s$. temperature.

that the anharmonicity of the translational mode along the molecular axis becomes considerable at high temperatures. The strong intrachain force field will not be so easily affected by temperature as the van der Waals intermolecular force field is.

\section{REFERENCES}

1. C. W. Bunn, Trans. Faraday Soc., 35, 482 (1939).

2. H. G. Kilian, Kolloid-Z. Z. Polymere, 183, 1 (1962).

3. H. G. Kilian, ibid., 185, 13 (1962).

4. Y. Aoki, A. Chiba and M. Kaneko, J. Phys. Soc. Japan, 27, 1579 (1969).

5. S. Kavesh and J. M. Schultz, J. Polym. Sci., $A-2,8,243$ (1970).

6. W. Ruland, Acta Cryst., 14, 1180 (1961).

7. T. Kitagawa and T. Miyazawa, Abstracts, SPSJ 16th Polymer Symposium, Fukuoka, October, 1967, p 230.

8. D. W. J. Cruickshank, Acta Cryst., 9, 747 (1956).

9. R. McWeeny, Acta Cryst., 4, 513 (1951).

10. Y. Nitta, Ed., "X-sen Kesshogaku (X-ray Crystallography”, Vol. II, Maruzen Co., Ltd., Tokyo, 1961, Chapter 3.

11. N. Kasai and M. Kakudo, Rep. Progr. Polym. Phys. Japan, 11, 145 (1968). 